Review Article

\title{
Dynamic Response of the Entrance Structure of an Elliptical Mountain Tunnel under the Action of SH Waves
}

\author{
Zhenyu Wang $\mathbb{D}^{1,2}$ Junsheng Yang, ${ }^{1}$ and Xinghua Wang ${ }^{1}$ \\ ${ }^{1}$ Central South University, Changsha 410075, China \\ ${ }^{2}$ Hunan University of Finance and Economics, ChangSha 410027, China
}

Correspondence should be addressed to Zhenyu Wang; adiwangzy@qq.com

Received 21 September 2021; Accepted 27 October 2021; Published 24 November 2021

Academic Editor: Yu Liang

Copyright (C) 2021 Zhenyu Wang et al. This is an open access article distributed under the Creative Commons Attribution License, which permits unrestricted use, distribution, and reproduction in any medium, provided the original work is properly cited.

Generally, the surrounding rock at the entrance of a mountain tunnel is loose, and the entrance has more slopes due to topography, which causes the tunnel entrance section to be easily destroyed under an earthquake. Based on the established slope model with a single free surface, this paper adopted the elastic wave theory to derive the analytical solution of the strain at the entrance of the mountain tunnel when the SH wave is incident perpendicularly to the bottom of the tunnel; besides, the factors affecting strain were also analyzed. The tangential strain curve at each point of the entrance section takes the centre of the elliptical tunnel as the centre of symmetry, forming symmetry between the left and right sides and mirror symmetry between the top and bottom sides. Then, large-scale shaking table model experiments were conducted to model the actual working conditions, and the correctness of the analytical solution was verified. The research can provide a theoretical reference for the seismic design of the entrance section of the high-speed railway tunnel and greatly improve the understanding of its seismic response.

\section{Introduction}

In China, the mountainous area accounts for more than $60 \%$, and most mountainous areas (such as the QinghaiTibet Plateau) are in the contact zone of the Eurasian Plate and the Indian Plate, where exists frequent seismic activity. With the continuous development of Chinese western development strategy, high-speed railways are also designed and constructed in this area; simultaneously, the anti-seismic problem of high-speed railway tunnels has attracted increasing attention. Moreover, the entrance section of the tunnel is an essential part of the tunnel and is prone to damage, making the entire tunnel unusable under the action of an earthquake. Due to the unique nature of high-speed railways, the tunnel is usually designed as a double-track single-hole similar to ellipse, with a relatively large span and a relatively large cross-sectional area, making the tunnel's entrance section more susceptible to earthquakes [1-5]. The method of studying the dynamic response of the tunnel entrance section includes theoretical analysis, numerical simulation, and shaking table model experiment. At present, researchers mainly adopt numerical simulation and shaking table model experiments, and the main research content is about the measures and effectiveness of anti-earthquake. During the research, the dynamic response of the entrance of the tunnel with a single cross-sectional structure is mainly considered, but the integrity of the tunnel and the three-dimensionality of the wave propagation are ignored [6-8]. The authors in $[9,10]$ only studied the dynamic response characteristics of the entrance section of the lower circular mountain tunnel under the effect of the SH wave and used the elliptical tunnel model to conduct shaking table tests to verify the analytical solution. The most significant difference between the tunnel entrance section and the tunnel body is that the tunnel entrance section has a free surface, while the tunnel body section can be regarded as a structure without an open surface. Based on the wave theory and the characteristics of the open surface at the tunnel entrance, the reflected wave was superimposed on the central axis of the tunnel to form a complex wave field, which makes the tunnel structure more complicated [11-18]. 
In this paper, the model of the entrance section of the mountain tunnel was simplified to a simple slope with a single surface, and it is believed that the structure of the tunnel does not affect wave propagation. Using the elastic wave propagation theory, when the $\mathrm{SH}$ wave was incident perpendicularly from the bottom of the tunnel, the dynamic stress along the axis of the elliptical mountain tunnel was derived. Then, the elliptical tunnel structure was regarded as a three-dimensional thin-walled shell to derive the analytical solution of the displacement of the tunnel structure under the action of SH wave. Finally, the shaking table model of the mountainous elliptical tunnel was conducted to verify the previous theoretically analyzed results and to summarize the deformation mechanism of the entrance section of the elliptical mountain tunnel.

\section{Theoretical Derivation}

To analyze the dynamic response of the entrance section of the elliptical mountain tunnel, it is first assumed that when the $\mathrm{SH}$ wave is incident perpendicularly from the bottom of the mountain, there is only the effect of the reflection of $\mathrm{SH}$ wave from the slope of the mountain, and the generated stress field at the axial of the tunnel is calculated. Then, while simplifying the tunnel into a three-dimensional thin-walled shell structure, the interaction between the surrounding rock and the tunnel is ignored to solve the displacement distribution of the tunnel structure under the action of the stress field.

2.1. Basic Assumptions. In the theoretical derivation process, the following assumptions are made:

(1) The ratio between the size of the surrounding rock mass and the size of the tunnel structure is so large that the influence of the existence of the tunnel structure on the dynamic response of the surrounding rock can be ignored.

(2) The link between the surrounding rock and the structure is tight, and there is no mutual displacement.

(3) The approximately elliptical tunnel of the high-speed railway is simplified to a standard elliptical structure.

(4) All structures are assumed to be a uniform elastic body.

2.2. Analytical Solution of Axial Displacement of Elliptical Model Tunnel. Figure 1 shows a single-sided slope model. According to the wave propagation theory, it can be known that $\mathrm{SH}$ waves are incident perpendicularly from the bottom, travel along $\mathrm{O} 1 \mathrm{~B}$ to point $\mathrm{B}$ on the slope, and then are reflected; the reflected waves propagated along BA to point $\mathrm{A}$, where it superimposed with the incident $\mathrm{SH}$ wave that directly travels along $\mathrm{O} 2 \mathrm{~A}$ (Figure 2). According to the wave propagation theory, the reflection angle $(\alpha)$ of the $\mathrm{SH}$ wave is equal to the slope angle $(\alpha)$ of a single-sided slope, the speed of the incident wave is equal to the reflected wave, and their amplitudes are equal [19].

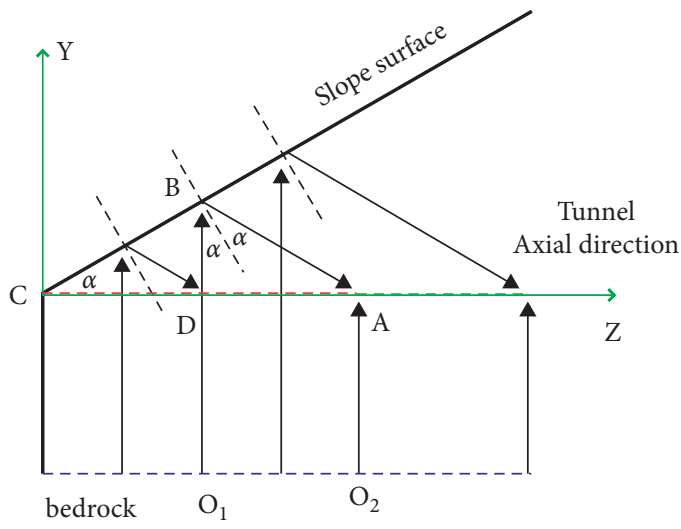

FIGURE 1: SH wave propagation model.

The function of the displacement of the incident wave is supposed to be

$$
u_{x}=A \times \sin \left[\frac{2 \pi}{L}\left(y-v_{s} t\right)\right],
$$

where $A$ is the amplitude, $L$ is the wavelength, and $V s$ is the shear wave velocity. If a single-sided slope is regarded as a free boundary, the function of the reflected wave displacement is

$$
u_{x}=A \times \sin \left[\frac{2 \pi}{L}\left(z \prime-v_{s} t^{\prime}\right)\right],
$$

where $t^{\prime}=t-t 0$, in which $t 0$ is the time when $\mathrm{SH}$ wave propagates from point $\mathrm{D}$ to point $\mathrm{A}$ through the slope reflection (see Figure 1).

In Figure 2, the origin of the coordinates is point $C$, the coordinate of point $\mathrm{A}$ is $(0, Z)$, and the delayed time of the reflected wave reaching point $\mathrm{A}$ is

$$
t_{0}=\frac{Z \sin (2 \alpha)}{v_{s}} \text {. }
$$

According to the research of Kuesel [20], the SH wave reflected by the mountain slope is equivalent to the following two waves.

The SH wave propagating along the tunnel axis $(Z$ direction) has a wavelength of $L / \cos (\pi / 2-2 \alpha)$, a wave speed of $v_{s} / \cos (\pi / 2-2 \alpha)$, and an amplitude of $A$. The SH wave propagating in the cross-sectional direction ( $Y$ direction) has a wavelength of $L / \sin (\pi / 2-2 \alpha)$, a wave speed of $v s / \sin (\pi /$ $2-2 \alpha$ ), and an amplitude of $A$. Therefore, the wavefield at point $A$ is the superposition of the above three waves (incident $\mathrm{SH}$ wave, $\mathrm{SH}$ wave propagating in the directions of axial, and cross section).

\subsubsection{The Strain of Tunnel Structure While Superimposing the} Wavefield. To solve the dynamic response of the tunnel structure, the tunnel is simplified as a thin-walled elliptical cylindrical shell, as shown in Figure 3. Because the thickness of the cross section of the thin-walled elliptic cylindrical shell is very small, only three strains of the structure, including axial strain, hoop strain, and tangential strain, need to be considered. 


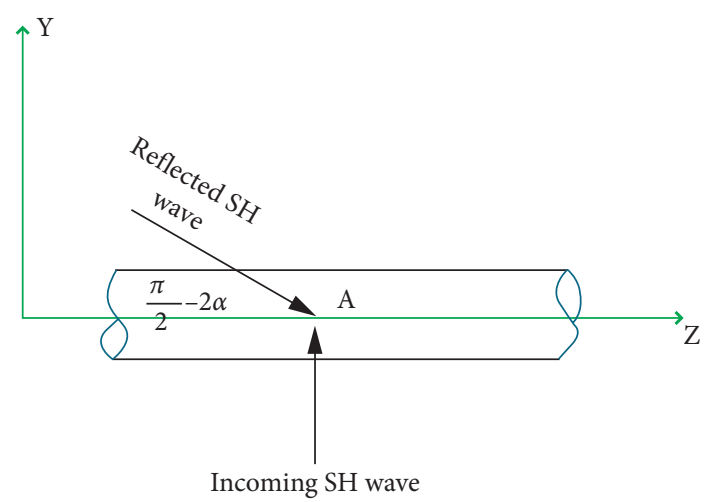

FIgURE 2: The illustration of the superposition of axial displacement at A point.

The calculation formulas of axial strain, hoop strain, and tangential strain are, respectively,

$$
\begin{aligned}
& \varepsilon_{\alpha}=\varepsilon_{z}=\frac{\partial u_{z}}{\partial z}, \\
& \varepsilon_{h}=\varepsilon_{\theta \theta}=\frac{1}{r(\theta)} \frac{\partial u_{\theta}}{\partial \theta}+\frac{u_{r \theta}}{r(\theta)}, \\
& \gamma=\gamma_{\theta z}=\frac{1}{r(\theta)} \frac{\partial u_{\theta}}{\partial \theta}+\frac{\partial u_{\theta}}{\partial z} .
\end{aligned}
$$
is

In the cylindrical coordinate system, the elliptic equation

$$
\begin{aligned}
\frac{r^{2}(\theta) \sin ^{2} \theta}{a^{2}}+\frac{r^{2}(\theta) \cos ^{2} \theta}{b^{2}} & =1, \\
r(\theta) & =\frac{a b}{\sqrt{b^{2} \sin ^{2} \theta+a^{2} \cos ^{2} \theta}},
\end{aligned}
$$

where $r(\theta)$ is the radius of the ellipse, $2 a$ and $2 b$ are the long and short axes of the elliptical tunnel, respectively, and $u z$, $u \theta$, and $u r$ are the components of displacement induced by the shear wave in the cylindrical coordinate system.

Therefore, under the impact of the superimposed displacement field, the total strain of the tunnel structure is equal to the superposition of the strain generated by each wave alone. At point A of the tunnel structure, the strain caused by the three waves is calculated as follows.

In the elliptic cylindrical coordinate system shown in Figure 4, the coordinates of point $X$ of the double-track single-hole structure of the high-speed railway are

$$
\begin{array}{r}
\frac{x^{2}(\theta)}{a^{2}}+\frac{y^{2}(\theta)}{b^{2}}=1, \\
\frac{x(\theta)^{\prime} x(\theta)}{a^{2}}+\frac{y(\theta)^{\prime} y(\theta)}{b^{2}}=0,
\end{array}
$$

where (' $\left.x(\theta),{ }^{\prime} y(\theta)\right) \perp\left(x(\theta) / a^{2}, y(\theta) / b^{2}\right)$.

$$
\left({ }^{\circ} x(\theta),{ }^{\prime} y(\theta)\right)\left(\frac{y(\theta)}{b^{2}},-\frac{x(\theta)}{a^{2}}\right)(\text { mark as } \overrightarrow{1}),
$$

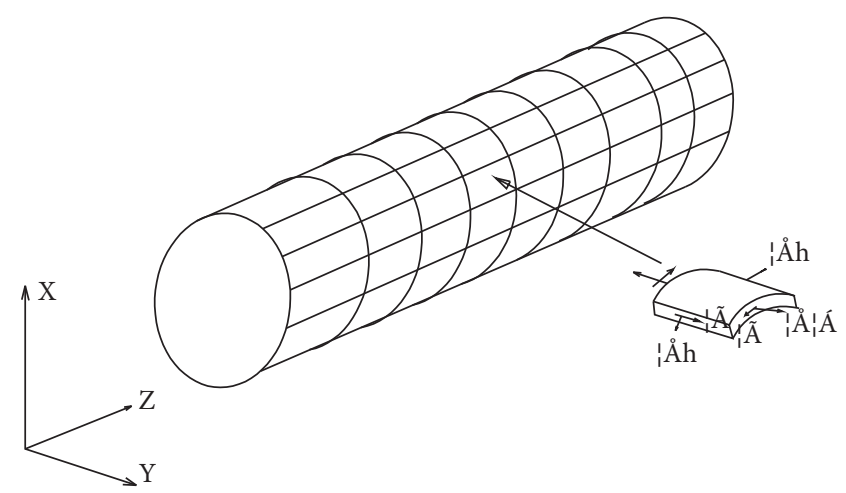

FIgURE 3: Tunnel lining strain of thin-walled elliptical cylindrical shell.

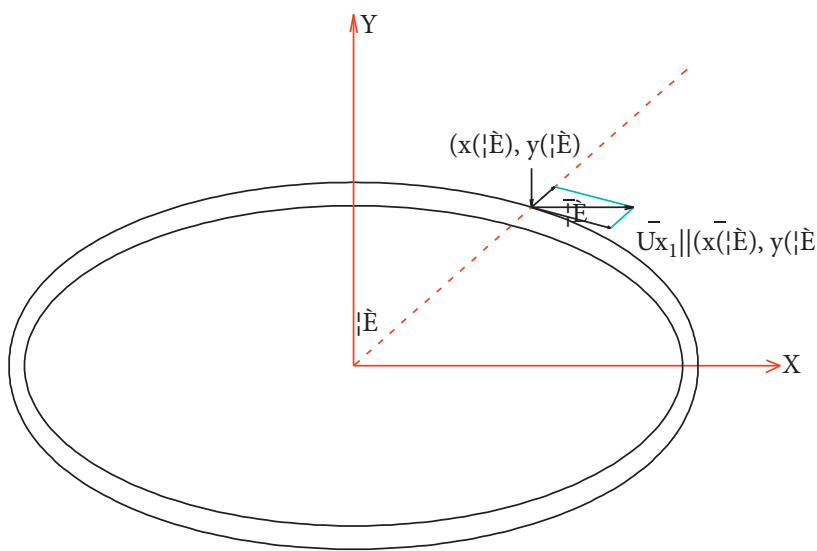

Figure 4: Figure ellip[[parms resize(1),pos(50,50),size(200,200),bgcol(156)]]ical cylindrical coordinates.

and therefore

$$
\begin{aligned}
& \cos \widetilde{\theta}=\frac{a \cos \theta}{\sqrt{b^{2} \sin ^{2} \theta+a^{2} \cos ^{2} \theta}}, \\
& \sin \widetilde{\theta}=\frac{b \sin \theta}{\sqrt{b^{2} \sin ^{2} \theta+a^{2} \cos ^{2} \theta}} .
\end{aligned}
$$

(1) Reflected SH Wave Propagating along the Tunnel Axis ( $Z$ Direction). The propagation of the reflected SH wave along the $Z$ direction in the $\mathrm{XOZ}$ plane is reflected by the displacement of the surrounding rock:

$$
u_{x}=A \times \sin \left[\frac{2 \pi}{L / \cos (\pi / 2-2 \alpha)}\left(z-\frac{v_{s} t_{1}^{\prime}}{\cos (\pi / 2-2 \alpha)}\right)\right] .
$$

According to the cylindrical coordinate system in Figure 4, the tunnel displacement can be decomposed into the tangential and radial displacement, and the corresponding functions are 


$$
\begin{aligned}
u_{x}= & A \times \sin \left[\frac{2 \pi}{L / \cos (\pi / 2-2 \alpha)}\left(z-\frac{v_{s} t_{1}^{\prime}}{\cos (\pi / 2-2 \alpha)}\right)\right] \\
u_{\theta}= & A \frac{a \cos \theta}{\sqrt{b^{2} \sin ^{2} \theta+a^{2} \cos ^{2} \theta}} \sin \\
& \cdot\left[\frac{2 \pi}{L / \cos (\pi / 2-2 \alpha)}\left(z-\frac{v_{s} t^{\prime}}{\cos (\pi / 2-2 \alpha)}\right)\right]
\end{aligned}
$$

By substituting formulas (12) and (13) into formulas (2) (4), the strain expression can be sorted and obtained as

$$
\begin{aligned}
& \varepsilon_{h}=\varepsilon_{\theta}=A \times A_{1} \times A_{2} \sin \left[A_{3}\left(Z-\frac{v_{s} t^{c}}{A_{4}}\right)\right], \\
& \gamma=A \times A_{5} \times A_{3} \cos \left[A_{3}\left(z-\frac{v_{s} t^{c}}{A_{2}}\right)\right], \\
& \varepsilon_{\alpha}=0
\end{aligned}
$$

where

$$
\begin{aligned}
& A_{1}=\frac{1}{(a b)}, \\
& A_{2}=\frac{\sin \theta\left(b^{3} \sin ^{2} \theta+a^{2} b \cos ^{2} \theta-a b^{2}\right)}{b^{2} \sin ^{2} \theta+a^{2} \cos ^{2} \theta}, \\
& A_{3}=\frac{2 \pi}{L / \cos (\pi / 2-2 \alpha)}, \\
& A_{4}=\cos \left(\frac{\pi}{2}-2 \alpha\right) \\
& A_{5}=\frac{a \cos \theta}{\sqrt{b^{2} \sin ^{2} \theta+a^{2} \cos ^{2} \theta}} .
\end{aligned}
$$

(2) Reflected SH Wave Propagating in the Y Direction. The displacement function corresponding to the reflected $\mathrm{SH}$ wave propagating in the $Y$ direction is

$$
u_{x}=A \times \sin \left[\frac{2 \pi}{L / \sin (\pi / 2-2 \alpha)}\left(-y-\frac{v_{s} t^{\prime}}{\sin (\pi / 2-2 \alpha)}\right)\right] .
$$

Similarly, the function of the tangential and radial displacement can be, respectively, obtained:

$$
\begin{aligned}
u_{r}= & A \frac{b \sin \theta}{\sqrt{b^{2} \sin ^{2} \theta+a^{2} \cos ^{2} \theta}} \\
& \cdot \sin \left[\frac{2 \pi}{L / \sin (\pi / 2-2 \alpha)}\left(-y-\frac{v_{s} t^{\prime}}{\sin (\pi / 2-2 \alpha)}\right)\right]
\end{aligned}
$$

$$
\begin{aligned}
u_{\theta}= & A \frac{a \cos \theta}{\sqrt{b^{2} \sin ^{2} \theta+a^{2} \cos ^{2} \theta}} \\
& \cdot \sin \left[\frac{2 \pi}{L / \sin (\pi / 2-2 \alpha)}\left(-y-\frac{v_{s} t^{\prime}}{\sin (\pi / 2-2 \alpha)}\right)\right] .
\end{aligned}
$$

By substituting formulas (17) and (18) into formulas (2) (4), the strain expression can be sorted and obtained as

$$
\begin{gathered}
\varepsilon_{h}=\varepsilon_{\theta}=A \times A_{1}\left\{A_{6} A_{7} \cos \left[A_{7}\left(-y-\frac{v_{s} t_{1}^{\prime}}{A_{8}}\right)\right]\right. \\
\left.+A_{2} \sin \left[A_{7}\left(-y-\frac{v_{s} t_{1}^{\prime}}{A_{8}}\right)\right] \gamma=\varepsilon_{\alpha}=0\right\},
\end{gathered}
$$

where

$$
\begin{aligned}
& A_{6}=\frac{a^{2} b^{3} \sin \theta \cos \theta}{\left(b^{2} \sin ^{2} \theta+a^{2} \cos ^{2} \theta\right)^{3 / 2}}, \\
& A_{7}=\frac{2 \pi}{L / \sin (\pi / 2-2 \alpha)}, \\
& A_{8}=\sin \left(\frac{\pi}{2}-2 \alpha\right) .
\end{aligned}
$$

(3) Incident SH Wave Propagating in the Y Direction. The displacement function corresponding to the incident $\mathrm{SH}$ wave propagating in the $Y$ direction is

$$
u_{x}=A \times \sin \left[\frac{2 \pi}{L}\left(y-v_{s} t\right)\right] .
$$

After decomposing the displacement function of the wave along with the tangential and radial directions, their functions can be obtained as

$$
\begin{aligned}
& u_{r}=A \frac{b \sin \theta}{\sqrt{b^{2} \sin ^{2} \theta+a^{2} \cos ^{2} \theta}} \sin \left[\frac{2 \pi}{L}\left(y-v_{s} t\right)\right], \\
& u_{\theta}=A \frac{a \cos \theta}{\sqrt{b^{2} \sin ^{2} \theta+a^{2} \cos ^{2} \theta}} \sin \left[\frac{2 \pi}{L}\left(y-v_{s} t\right)\right] .
\end{aligned}
$$

By substituting formulas (22) and (23) into formulas (2) to (4), the strain expression can be sorted and obtained as

$$
\left.\begin{array}{rl}
\varepsilon_{h} & =A_{1}\left\{A_{2} \sin \left[A_{9}\left(y-v_{s} t\right)\right]-A_{9} A_{6} \cos \left[A_{9}\left(y-v_{s} t\right)\right]\right\}, \\
\gamma & =\varepsilon=0,
\end{array}\right\}
$$

where

$$
A_{9}=\frac{2 \pi}{L}
$$

After superimposing the tunnel structure strain caused by the above three $\mathrm{SH}$ waves and eliminating the time term $t$ ', the total strain of the tunnel structure can be obtained: 


$$
\begin{aligned}
\mathcal{E}_{\alpha}= & 0 \\
\gamma= & A \times A_{5} \times A_{3} \cos \left[A_{3}\left(2 z-\frac{v_{s} t}{A_{4}}\right)\right], \\
\mathcal{E}_{h}= & A_{1}\left(A_{2}\left\{\sin \left[A_{3}\left(2 Z-\frac{v_{s} t}{A_{4}}\right)\right]+\sin \left[A_{7}\left(z \operatorname{tg} 2 \alpha-y-\frac{v_{s} t}{A_{8}}\right)\right]+\sin \left[A_{9}\left(y-v_{s} t\right)\right]\right\},\right. \\
& \left.+A_{6}\left\{B_{3} \cos \left[A_{7}\left(z \operatorname{tg} 2 \alpha-y-\frac{v_{s} t}{A_{8}}\right)\right]-A_{9} \cos \left[A_{9}\left(y-v_{s} t\right)\right]\right\}\right) .
\end{aligned}
$$

It can be seen from formula (26) that under the action of the $\mathrm{SH}$ wave incident vertically at the bottom, the axial strain of the tunnel structure is 0 , the hoop strain is generated by the $\mathrm{SH}$ wave propagating along the $Y$-axis (parallel cross section), and the tangential strain is generated by $\mathrm{SH}$ waves propagating in the $Z$-axis direction (axial direction). The above three equations are the expressions of the total strain of the entrance section of the elliptical mountain tunnel. When the tunnel structure is a circle, which means the long and short axes are equal, formula (26) will degenerate into the form in literature [9].

\section{Analysis of the Influencing Factors of the Dynamic Response of the Entrance Section}

To analyze the influence of various factors on the dynamic response of the entrance section of the mountain tunnel, firstly, the displacement function of the incident wave is assumed:

$$
f(t)=A \sin (2 \pi f t),
$$

where the frequency $f$ is $2 \mathrm{~Hz}$ and the amplitude $A$ is $0.3 \mathrm{~m}$. According to formulas (26) and (27), the dynamic strain of the elliptical tunnel structure can be calculated.

The parameters are as follows: the long axis of the elliptical tunnel is $2 a=11.2 \mathrm{~m}$, the short axis $2 b=8.6 \mathrm{~m}$, and the thickness is $0.48 \mathrm{~m}$. The parameters of the surrounding rock and lining are shown in Table 1, and the studied scope in the axial direction of the tunnel is taken as $100 \mathrm{~m}$ $(z=100 \mathrm{~m})$, and the portal angle is set to $70^{\circ}$ to facilitate comparison with the shaking table model test results.

Figure 5 shows the tangential strain curve of each position of the mountain tunnel opening section when the $\mathrm{SH}$ wave is incident from the bottom of the tunnel; it can be known that all the tangential strain curves are similar to a sine curve, and when the slope is $45^{\circ}$, the amplitude of the strain curve is the largest, which appears at the arch base and the vault, and their absolute value is equal to $\mathcal{E}=6.283 \varepsilon \mu$, but the sign is opposite. The strain at the left (right) arch waist is zero, and the tangential strain at each position of the tunnel entrance section is mirror-symmetric with the axis of the tunnel as the centre; when the slope of the mountain slope is greater than or equal to $60^{\circ}$, the tangential strain curve of each position of the opening section begins to reverse, and the largest absolute value of the strain still appears at the vault and arch bottom of the tunnel, but when the slope is $89^{\circ}$, the tangential strain at each position of the tunnel opening section is almost zero.

Figure 6 shows the relationship between the tangential strain at each position of the tunnel opening section and the slope of the mountain slope; it can be seen that when the slope is $45^{\circ}$, the absolute value of the tangential strain at each position of the tunnel opening section is the largest; as the slope of the slope increases, this absolute value decreases, and the tangential strain decreases to 0 at about $60^{\circ}$. With the continuous increase of the slope, the absolute value of the tangential strain keeps increasing and reaches the maximum when the slope is $70^{\circ}$. When the slope continues to increase, the absolute value of the tangential strain begins to decrease and tends to 0 when the slope is $89^{\circ}$. Besides, it can also be seen that the tangential strain curve at each position of the mountain tunnel entrance section is symmetrical with the centre of the elliptical tunnel as the centre; combining Figures 5 and 6, it can also be known that this tangential strain is mirror-symmetric at the top and bottom sides.

Figure 7 shows the relationship between the tangential strain at each position of the entrance section and the distance from the entrance, which further verifies the symmetry of the tangential strain curve; in the range of $100 \mathrm{~m}$ from the cave opening, each tangential strain curve undergone 6 times of phase inversion. Figure 8 shows the relationship between the tangential strain at the right shoulder of the tunnel entrance and the ratio of the long axis to the short axis $(a / b)$; it can be seen that as the value of $\mathrm{a} / \mathrm{b}$ increases, the tangential strain at the right shoulder of the tunnel also increases, but the increment varies with the slope of the mountain slope. Only when the slope is $45^{\circ}$, the strain is positive; at this time, the increment is the largest (from $4.649 \varepsilon \mu$ to $5.620 \varepsilon \mu$ ), which is followed by the slope of $70^{\circ}$; when the slope is $89^{\circ}$, the tangential strain increases from $-0.095 \varepsilon \mu$ to $-0.114 \varepsilon \mu$.

\section{The Preparation of a Large-Scale Shaking Table Test Model}

To verify the theoretical analysis result, a large-scale shaking table experiment was designed to model the on-site working condition. To objectively and truly reflect the characteristics of the prototype, a similar design is required. Other physical parameters are shown in Table 2. 
TABLE 1: Mechanical parameters of lining and rock materials.

\begin{tabular}{lcccc}
\hline & & Bulk density, $\gamma\left(\mathrm{kN} / \mathrm{m}^{3}\right)$ & Elastic modulus, $E(\mathrm{GPa})$ & Poisson's ratio \\
\hline \multirow{2}{*}{ Wall rock } & Soft rock & 17.09 & 0.65 & 0.22 \\
Tunnel lining & Hard rock & 22.3 & $31.5(23.4 \mathrm{MPa})$ & 0.23 \\
\hline
\end{tabular}

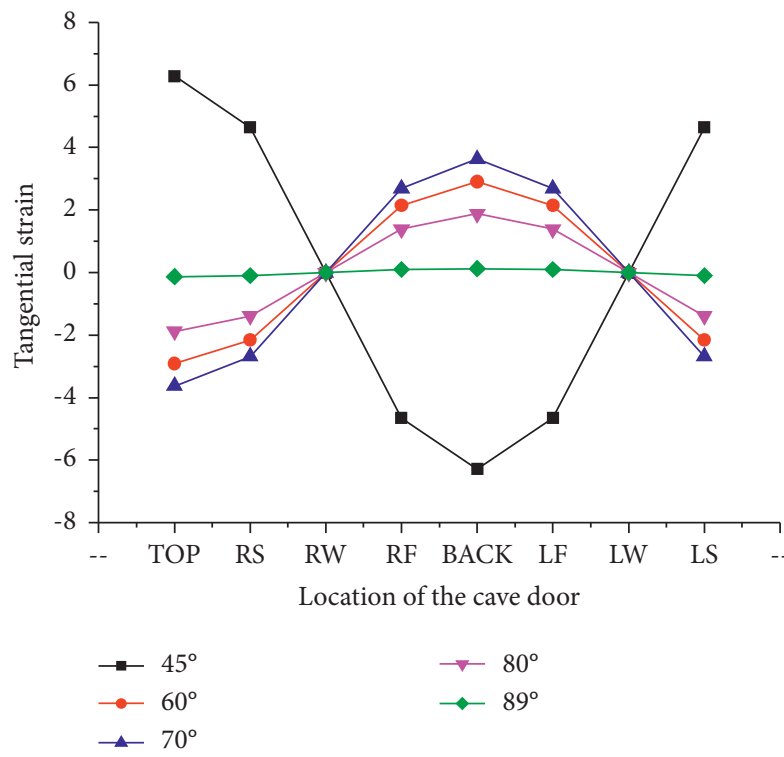

Figure 5: Tangential strain of opening section.

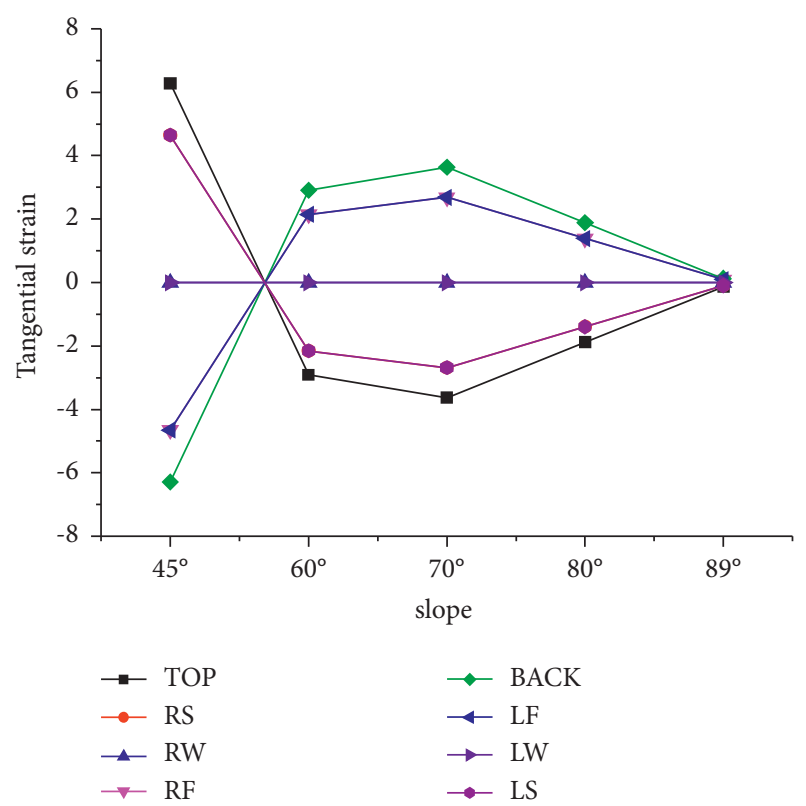

FIgURE 6: The influence of mountain slope.

4.1. The Preparation of the Elliptical Tunnel Model and Monitoring Plan. As shown in Figures 9 and 10, considering the size of the vibrating table and its ultimate bearing capacity, the size of the model box is set as

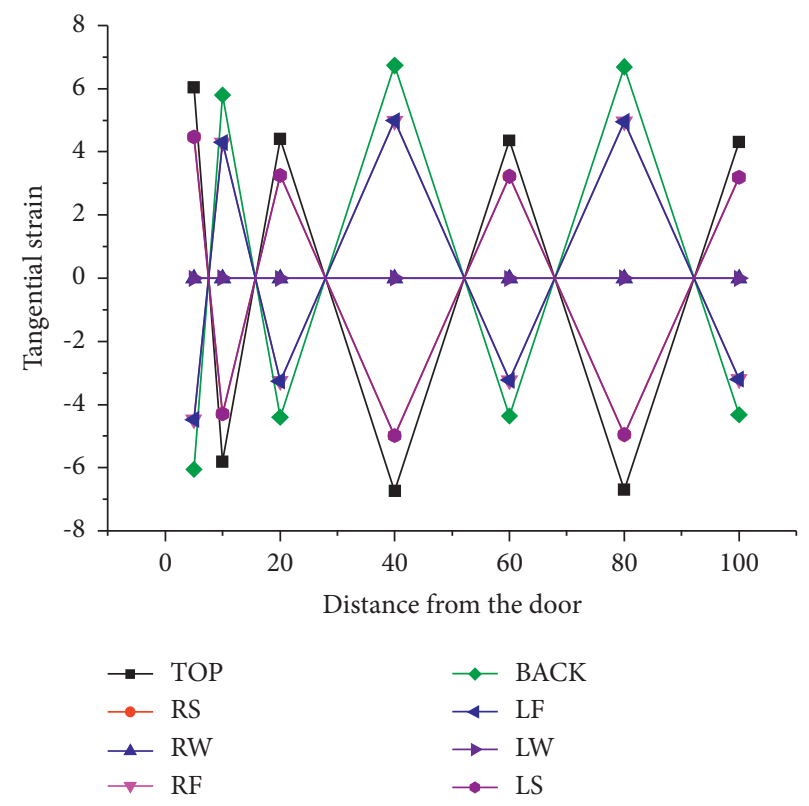

Figure 7: The influence of tunnel opening distance.

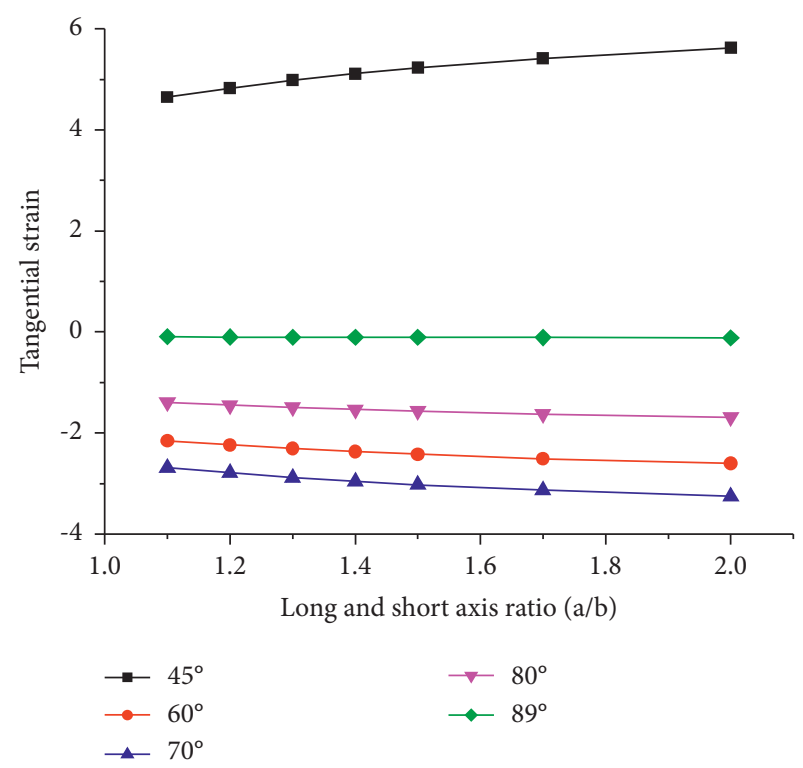

FIgURE 8: The influence of the long and short axes.

$9300 \mathrm{~mm} \quad$ (length) $\times 3700 \mathrm{~mm} \quad$ (width) $\times 2500 \mathrm{~mm}$ (height), and the total weight is 3.8 tons.

In the tunnel model test, along the axial direction of the tunnel, from left to the right, they are mainly arranged at the 
TABle 2: Physical dimension and similarity relation of model test.

\begin{tabular}{lcc}
\hline Physical quantity & Dimension & Similarity ratio \\
\hline Length & $C_{l}$ & $1 / 8$ \\
Mass density & $C \rho$ & $1 / 1$ \\
Elastic modulus & $C_{t}=C_{l}^{1 / 2}$ & $1 / 1$ \\
Time & - & 0.353 \\
Poisson's ratio & $C \sigma=\mathrm{CE} \varepsilon$ & 1 \\
Stress & $C \sigma=\mathrm{CE} C \varepsilon$ & 1 \\
Cohesion & $C_{\gamma}=C_{E} C_{t}$ & 1 \\
Coefficient of viscosity & - & 0.353 \\
Internal friction angle & $C \varepsilon$ & 1 \\
Strain & $C_{\mu}$ & 1 \\
Displacement & $C_{v}=C_{l} C_{t}^{-1}$ & 0.125 \\
Velocity & $C_{\alpha}=C_{l} C_{t}^{-2}$ & 0.353 \\
Acceleration & $C_{F}=C_{\gamma} C_{l}^{3}$ & 1 \\
Strength & $C_{N}=C_{t}^{-1}$ & 15625 \\
Frequency & $C_{\varsigma}=C_{\gamma} C_{l}^{2} C_{t}$ & 2.828 \\
Damping coefficient &
\end{tabular}

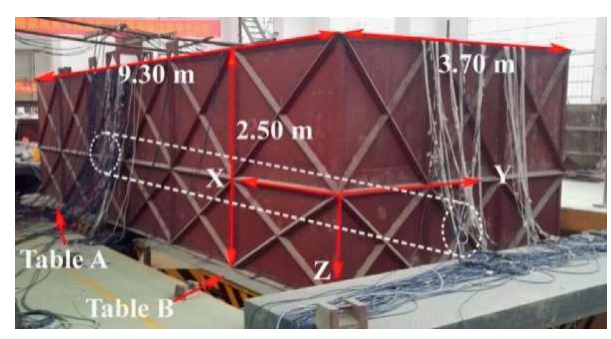

Figure 9: Outside of the model box.

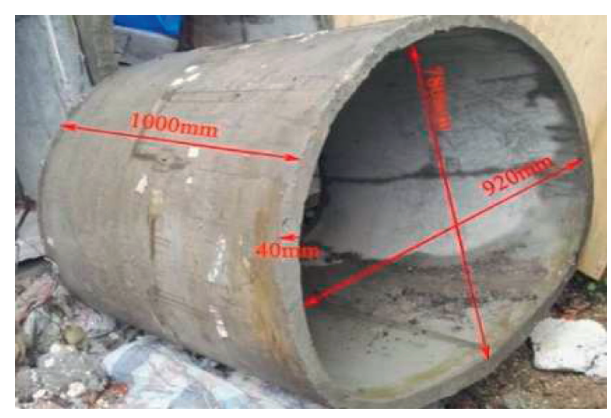

Figure 10: Oval tunnel lining model.

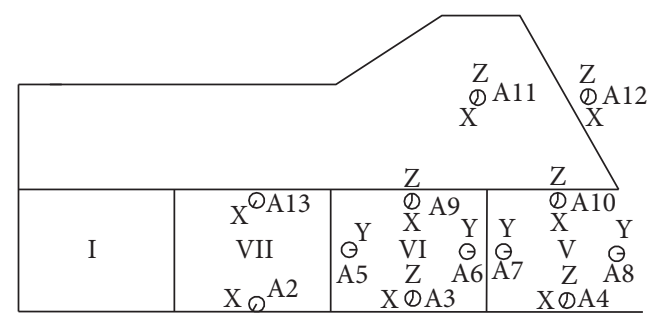

๑ X, Y Two-directional accelerometer

$\otimes \mathrm{X}, \mathrm{Y}, \mathrm{Z}$ Three-directional accelerometer

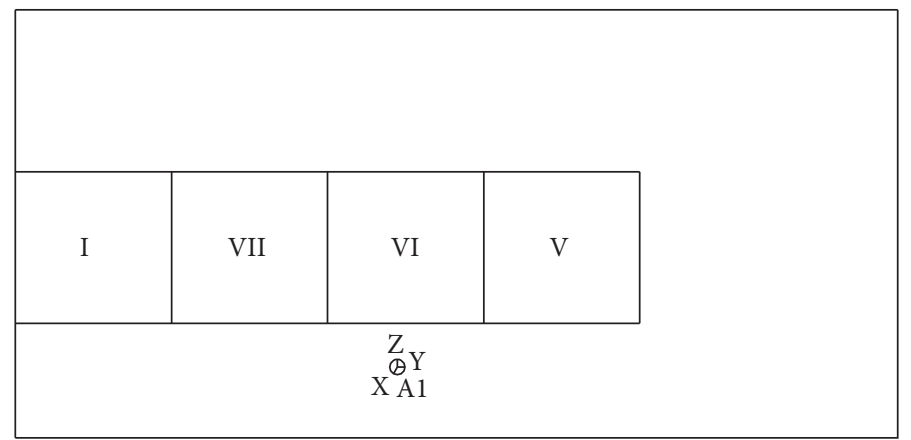

FIGURE 11: Schematic diagram of the longitudinal section and bottom of the elliptical tunnel opening section. 


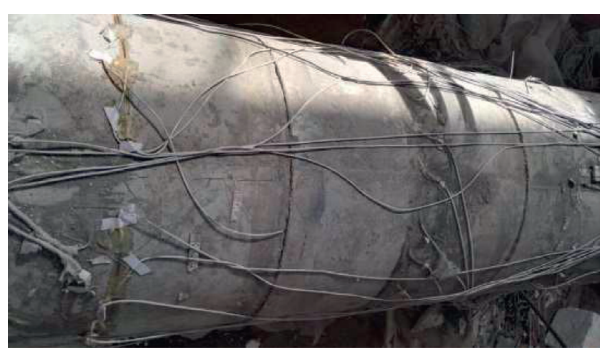

FIgURE 12: External view of the oval tunnel opening.

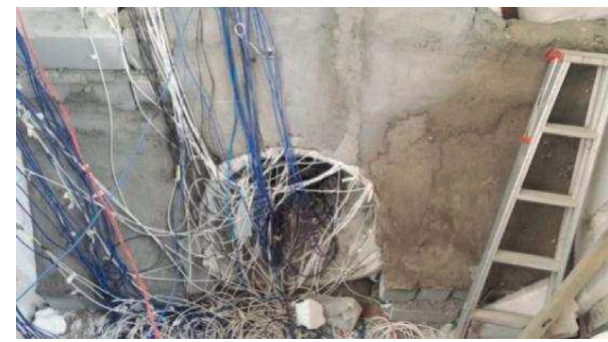

Figure 13: Oval tunnel opening map.

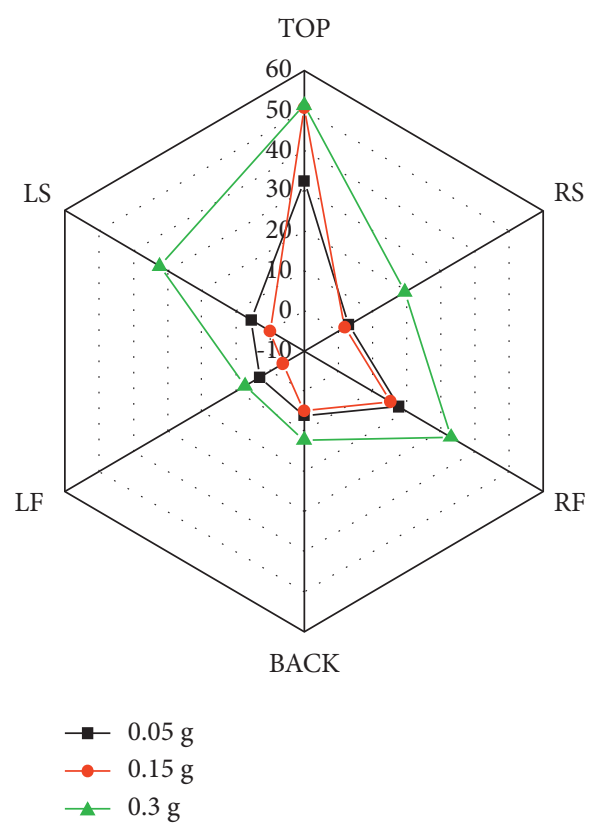

Figure 14: Circumferential strain inside the tunnel portal $(4 \mathrm{~Hz})$.

positions of VII-VII, VI-VI, and IX-IX and the periphery of the tunnel, as shown in Figures 11-13.

4.2. Analysis of Model Test Results. For different seismic intensities, the strain development of the tunnel is the same. The change of the hoop strain of the tunnel entrance section is as shown in Figures 14 and 15.

It can be known that when a sinusoidal seismic wave is input, the most dangerous parts of the tunnel are the right arch foot and the vault. When the seismic frequency is $4 \mathrm{~Hz}$,

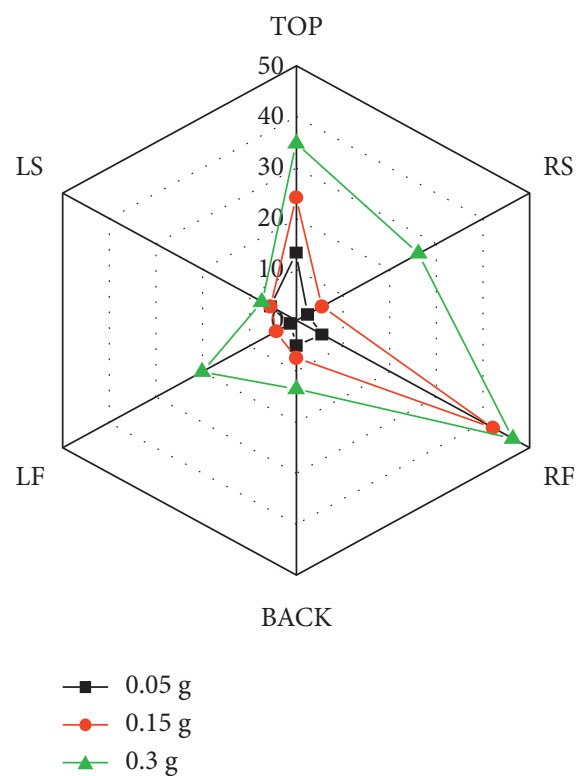

Figure 15: Circumferential strain inside the tunnel portal $(7.237 \mathrm{~Hz})$.

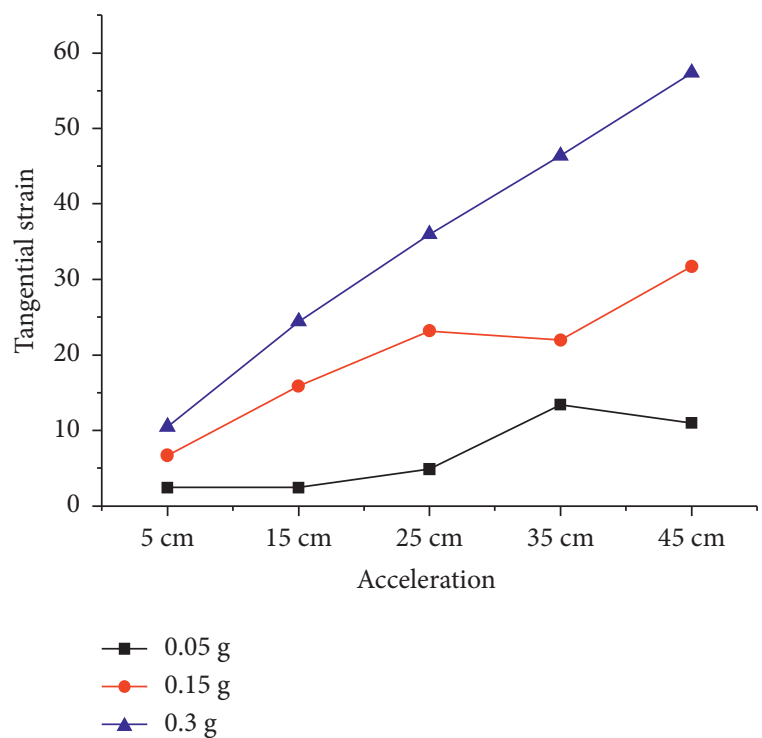

FIGURE 16: Longitudinal strain curve of vault exterior $(6.383 \mathrm{~Hz})$.

the maximum strain appears on the vault (acceleration is $0.3 \mathrm{~g}$ and strain is $51.27 \mu \varepsilon$ ); when the seismic frequency is $7.237 \mathrm{~Hz}$, the maximum strain appears on the right arch foot (acceleration is $0.3 \mathrm{~g}$ and strain is $46.39 \mu \varepsilon$ ).

Figures 16 and 17 show the longitudinal strain of the tunnel vault outside when the sinusoidal seismic wave is input, and it can be seen that as the seismic acceleration increased, the strain of the tunnel also increases; this variation has nothing to do with the frequency of the input seismic wave. When the frequency of the seismic wave is relatively small $(6.38 \mathrm{~Hz})$, the strain of the tunnel increases with the increase of the distance from the opening; when the frequency is relatively large $(13 \mathrm{~Hz})$, the strain of the tunnel 


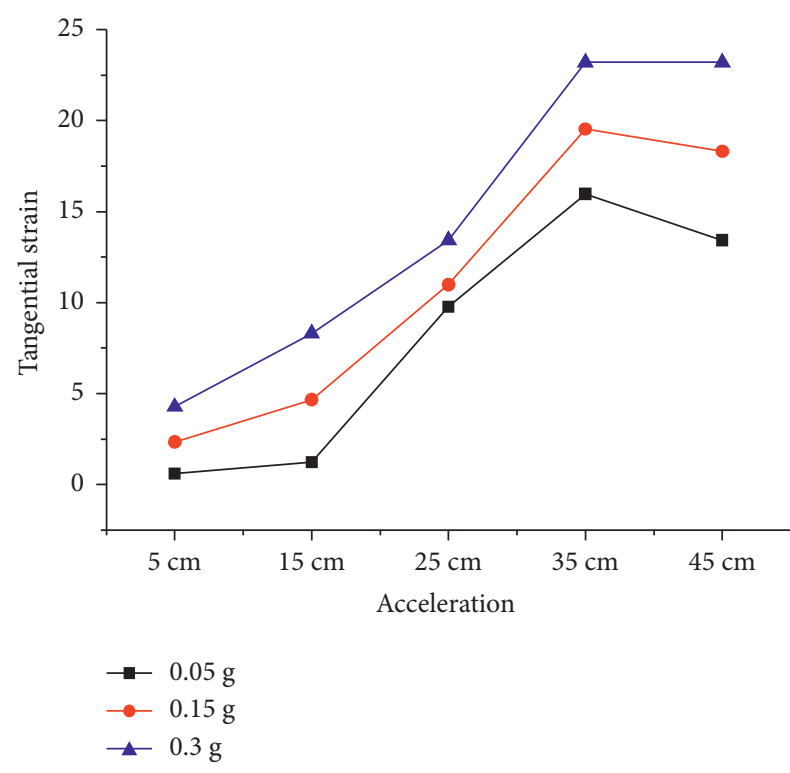

Figure 17: Longitudinal strain curve of vault exterior $(13 \mathrm{~Hz})$.

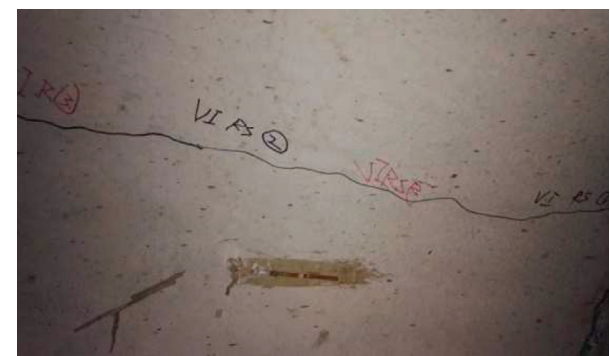

FIGURE 18: Destruction diagram of the right spandrel at the entrance of the tunnel.

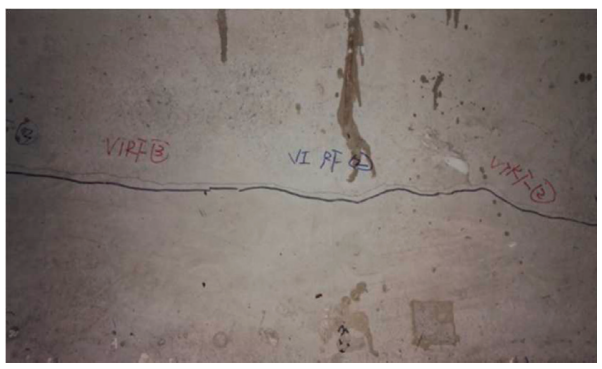

FIGURE 19: Destruction diagram of the right arch foot of the tunnel entrance section.

still increases with the increase of the distance from the opening, but the most dangerous section is $35 \mathrm{~cm}$ from the entrance of the cave. Under the action of the axial seismic wave, horizontal shear deformation occurs along the axial direction of the tunnel, and a large rigid displacement appears at the tunnel entrance, which makes the right shoulder and right foot easily undergo destruction (Figures 18 and 19).
The test results above preliminarily verify the theoretical analysis results.

\section{Conclusions}

In this paper, under the effect of the $\mathrm{SH}$ wave incident from the bottom of the tunnel, the strain at the entrance of the elliptical mountain tunnel was simplified and deduced theoretically; at the same time, a large-scale shaking table model test was carried out to verify the analytical results.

(1) By simplifying the slope of the mountain into a slope with a single surface, when the $\mathrm{SH}$ wave is incident vertically at the bottom, an analytical solution for the deformation at the entrance of the elliptical mountain tunnel is obtained. By analyzing the parameter, it is known that when the sinusoidal SH wave is input, the curve of tangential strain at the tunnel entrance section is symmetric, with the centre of the tunnel as the centre. The strain curves at the left and right of the tunnel are symmetrical, while the strain curves at the top and bottom of the tunnel are mirror-symmetrical, and their values are equal with opposite signs.

(2) Through parameter analysis, it can be confirmed that as the ratio of the long axis to the short axis of the elliptical tunnel increases, the tangential strain at the right shoulder of the tunnel portal section increases slowly, and the magnitude of the increment varies with the slope of the mountain slope. When the slope is $45^{\circ}$, the strain is positive, and other slopes are negative; when the slope is $45^{\circ}$, the increment is the largest $(0.971 \varepsilon \mu)$, followed by $70^{\circ}$; when the slope is $89^{\circ}$, the tangential strain increases from $-0.095 \varepsilon \mu$ to $-0.114 \varepsilon \mu$, which only increases by $0.019 \varepsilon \mu$.

(3) With the large-scale shaking table model, considering the seismic wave reflection effect, the rationality of the theoretical model of the single-surface slope at the tunnel entrance is verified. It is proved that when the frequency of the earthquake is different, the most dangerous position is also different. For example, when the frequency is $4 \mathrm{~Hz}$, the most dangerous position is the vault (the strain is $51.27 \mu \varepsilon$ and the acceleration is $0.3 \mathrm{~g}$ ), and when the frequency is $7.237 \mathrm{~Hz}$, the maximum strain appears at the right arch (acceleration is $0.3 \mathrm{~g}$ and strain is $46.39 \mu \varepsilon$ ), which is also confirmed by the failure results of the test model.

(4) The dynamic response is regarded as the superposition of the dynamic response in the direction of the tunnel cross section and longitude; the cross section is prone to shear deformation, making the shoulders and feet be the vulnerable parts during seismic. This phenomenon increases slowly along the axial direction, which is caused by $\mathrm{SH}$ waves propagating parallel to the cross section of the tunnel structure. 


\section{Data Availability}

The data used to support the findings of this study are available from the corresponding author upon request.

\section{Conflicts of Interest}

The authors declare that they have no conflicts of interest regarding the publication of this paper.

\section{Acknowledgments}

This study was supported by the Key Project of China Railway (2014G010-G).

\section{References}

[1] M. Gan, "Investigation of earthquake diseases on tunnel engineering," Investigation and Study of Earthquake Disease on Hanchuan Earthquake Engineering, vol. 6, 2009.

[2] Y. Yu, Seismic Damage Mechanism of Secondary Lining of Mountain tunnel, China Earthquake Administration, Harbin, China, 2013.

[3] Li Lin, Research on Seismic Response and Seismatic Design of Tunnel Grossing fault, Southwest Jiaotong University, Chengdu, China, 2009.

[4] C.. Jiao, Analysis on Earthquake Dynamic Response of Compound Tunnel anchorage of Suspension bridge, Southwest Jiaotong University, Chengdu, China, 2008.

[5] S. Huang, Research of Failure Mechanism and Seismic Measure for Underground Engineering under High Intensity earthquake, Wuhan Institute of Rock \&Soil Mechanics Chinese Academy of Sciences, Wuhan, China, 2020.

[6] M. Yang, Study on the Influence Elements of Seismic Dynamic Response of the portal of Mountain Tunnel in High Intensity Seismic zone, Beijing Jiaotng University, Beijing, China, 2011.

[7] X. Chao, Research on Dynamic Response at portal Section of Mountain Tunnel under Intense Earthquake and Shock Absorption measeure, Lanzhou Jiaotong University, Lanzhou, China, 2013.

[8] Ke. Tang, Study on the Influence Elements of Seismic Dynamic Response of portal of Mountain tunnel, Southwest Jiaotong University, Chengdu, China, 2006.

[9] S. Hou and L. Tao, "Dynamic response of portal section of mountain tunnel under seismic SH wave action," Chinese Journal of Rock Mechanics and Engineering, vol. 34, no. 2, pp. 340-348, 2015.

[10] T. Lian-jin and S. Hou, "Large-scale shaking table test for dynamic response in the portal section of mountain tunnel with different gradients of upward slope," Rock and Soil Mechanics, vol. 35, no. S1, pp. 91-98, 2014.

[11] V. W. Lee and J. Karl, "Diffraction of SV waves by underground, circular, cylindrical cavities," Soil Dynamics and Earthquake Engineering, vol. 11, no. 8, pp. 445-456, 1992.

[12] V. W. Lee and J. Karl, "Diffraction of elastic plane P waves by circular, underground unlined tunnels[J]," European Earthquake Engineering, vol. 6, no. 1, pp. 29-36, 1993.

[13] V. W. Lee, S. Chen, and I. R. Hsu, "Antiplane diffraction from canyon above subsurface unlined tunnel," Journal of Engineering Mechanics, vol. 125, no. 6, pp. 668-675, 1999.

[14] C. A. Davis, V. W. Lee, and J. P. Bardet, "Transverse response of underground cavities and pipes to incident SV waves," Earthquake Engineering \& Structural Dynamics, vol. 30, no. 3, pp. 383-410, 2001.
[15] V. W. Lee, L. Hao, and L. Jianwen, "Diffraction of anti-plane SH waves by a semi-circular cylindrical hill with an inside concentric semi-circular tunnel," Earthquake Engineering and Engineering Vibration, vol. 3, no. 2, pp. 249-262, 2004.

[16] C. Yi, P. Zhang, D. Johansson, and U. Nyberg, "Dynamic response of a circular lined tunnel with an imperfect interface subjected to cylindrical P-waves," Computers and Geotechnics, vol. 55, pp. 165-171, 2014.

[17] C. P. Yi, W. b. Lu, P. Zhang, D. Johansson, and U. Nyberg, "Effect of imperfect interface on the dynamic response of a circular lined tunnel impacted by plane P-waves," Tunnelling and Underground Space Technology, vol. 51, pp. 68-74, 2016.

[18] H. F. Kara, "A note on response of tunnels to incident SHwaves near hillsides," Soil Dynamics and Earthquake Engineering, vol. 90, pp. 138-146, 2016.

[19] H. Dai, Mechanics of Elasticity, Vol. 8, Hunan University Press, , Changsha, China, 2014.

[20] T. R. Kuesel, "Earthquake design criteria for subways," Journal of the Structural Division, vol. 95, no. 6, pp. 1213-1231, 1969. 